\title{
Remarques sur la création plurilingue chez Fernando Pessoa
}

João Dionísio

\section{(2) OpenEdition}

10 Journals

Édition électronique

URL : http://journals.openedition.org/genesis/2858

DOI : 10.4000/genesis.2858

ISSN : 2268-1590

Éditeur :

Presses universitaires de Paris Sorbonne (PUPS), Société internationale de génétique artistique littéraire et scientifique (SIGALES)

Édition imprimée

Date de publication : 4 juin 2018

Pagination : 93-102

ISBN : 979-10-231-0604-6

ISSN : 1167-5101

\section{Référence électronique}

João Dionísio, «Remarques sur la création plurilingue chez Fernando Pessoa », Genesis [En ligne], 46 | 2018, mis en ligne le 01 juin 2019, consulté le 23 septembre 2020. URL : http://

journals.openedition.org/genesis/2858; DOI : https://doi.org/10.4000/genesis.2858 


\title{
Remarques sur la création plurilingue chez Fernando Pessoa*
}

\author{
João Dionísio
}

L 'écrivain portugais Fernando Pessoa (1888-1935) mâ̂trisait plusieurs langues étrangères comme l'anglais, le français, l'allemand, l'espagnol, le latin et le grec ancien. Alors qu'au début du $\mathrm{XX}^{\mathrm{e}}$ siècle le Portugal vivait encore sous l'influence de la langue et de la culture françaises, ses écrits se démarquaient par une forte dimension anglophone ${ }^{1}$. La formation scolaire de Pessoa en Afrique du Sud a sans doute été un facteur crucial qui l'a amené au désir de devenir d'abord un écrivain de langue anglaise. Cette intention s'est matérialisée, entre autres, dans les opuscules de poésie anglaise qui ont paru en 1918 et 1921 à Lisbonne, dans la publication du poème «Meantime» dans la revue Athenaeum (en 1920), ainsi que dans le cycle poétique The Mad Fiddler, soumis à plusieurs maisons d'édition anglaises. Par conséquent, l'idée de Pessoa de s'affirmer comme écrivain portugais est postérieure à cette première intention d'entrer dans le monde des lettres anglaises. D'autre part, Pessoa a appris le français à l'école en Afrique du Sud, bénéficiant de la compétence francophone de sa mère. Ainsi, bien qu'il maîtrisât la langue française de manière moins virtuose que l'anglais, il s'est également servi du français pour sa création littéraire. Quant aux autres langues, à des degrés différents, elles paraissent être surtout objet d'apprentissage scolaire et/ou autodidacte plus ou moins occasionnel ${ }^{2}$. Il n'est donc pas surprenant de trouver dans le fonds Pessoa de la Bibliothèque nationale $\mathrm{du}$ Portugal (Esp. E 3), qui conserve la plupart des documents de travail du poète, dont une grande partie est restée inédite, des documents qui attestent d'expériences poétiques non seulement en portugais mais aussi en anglais et en français ${ }^{3}$, ainsi que d'une intense activité de traduction ${ }^{4}$. L'exploration des matériaux plurilingues dans le fonds Fernando Pessoa

(*) Une version préliminaire de cet article a fait l'objet de deux communications, «Fernando Pessoa's English poems and poetry written in English» et «Pessoa bilingue», présentées respectivement dans le cadre du colloque A poesia inglesa de Fernando Pessoa. Tributo a Georg Rudolf Lind, dirigé par Patricio Ferrari, Lisboa, Casa Fernando Pessoa, 2014 ; et au congrès international Fernando Pessoa, org. Antonio Cardiello, Mariana Gray de Castro, Pedro Sepúlveda et Clara Riso, Lisboa, Casa Fernando Pessoa/Fondation Calouste Gulbenkian, en février 2017. Je voudrais remercier Ivo Castro pour la lecture de cet article; mes remerciements vont aussi à Luís Prista, à la Biblioteca Nacional de Portugal et à la Casa Fernando Pessoa. Les révisions linguistiques sont de Nadia Gilardi. Cet essai a bénéficié de la lecture et des commentaires d'Olga Anokhina et d'Emilio Sciarrino.

1. À propos de la dépendance portugaise face à la France, le romancier Eça de Queirós a déclaré : «Le Portugal est un pays traduit du français en argot» (cité dans Carlos Reis, «Mode et transgression : Eça de Queirós et les “modes parisiennes”», Études françaises, vol. XX, n² 2, automne 1984, p. 96). 2. À propos des compétences linguistiques de Fernando Pessoa, voir par exemple, Arnaldo Saraiva, «Fernando Pessoa, théoricien de la traduction», dans Pascal Dethurens et Maria Alzira Seixo (dir.), Pessoa : unité, diversité, obliquité, Paris, Christian Bourgois, 2000, p. 382-384. Une bonne introduction à la bibliographie critique sur le bilinguisme anglais-portugais chez Pessoa se trouve en Patricia Ferreira, «Entre duas pátrias: o bilinguismo de Fernando Pessoa », Pessoa Plural. Revista de Estudos Pessoanos/A Journal of Fernando Pessoa Studies 6, Outono-Fall 2014, p. 60-78.

3. Voir José Blanco, Pessoana, I - Bibliografia passiva, selectiva e temática, II - Índices, Lisboa, Assírio \& Alvim, 2008 (en particulier II, p. 58, les sections 05.03.01.01 et suivantes, sur le bilinguisme, la langue française, la langue anglaise et le plurilinguisme); Fernando Pessoa, Poemas Ingleses, I, éd. João Dionísio, Lisboa, Imprensa Nacional - Casa da Moeda, 1993 ; II, éd. João Dionísio, Lisboa, Imprensa Nacional - Casa da Moeda, 1997 ; III, éd. Marcus Angioni et Fernando Gomes, Lisboa, Imprensa Nacional - Casa da Moeda, 1999; Patricio Ferrari, «Genetic Criticism and the Relevance of Metrics in Editing Pessoa's Poetry », Pessoa Plural, nº 2, 2012, p. 1-57; Patricio Ferrari et Jerónimo Pizarro (dir.), Fernando Pessoa as English Reader and Writer. Portuguese Literary \& Cultural Studies, $\mathrm{n}^{\circ} 28$, Spring 2015, University of Massachusetts, Dartmouth, Tagus Press; Fernando Pessoa, Obras de Jean Seul de Méluret, éd. Rita Patrício et Jerónimo Pizarro, Lisboa, Imprensa Nacional-Casa da Moeda, 2006; Fernando Pessoa, Poèmes français, éd. Patricio Ferrari avec la collab. de Patrick Quillier, Paris, La Différence, 2014.

4. À ce sujet voir Arnaldo Saraiva, Fernando Pessoa Poeta - Tradutor de Poetas. Os poemas traduzidos e o respectivo original, Porto, Lello Editores, 1996. 


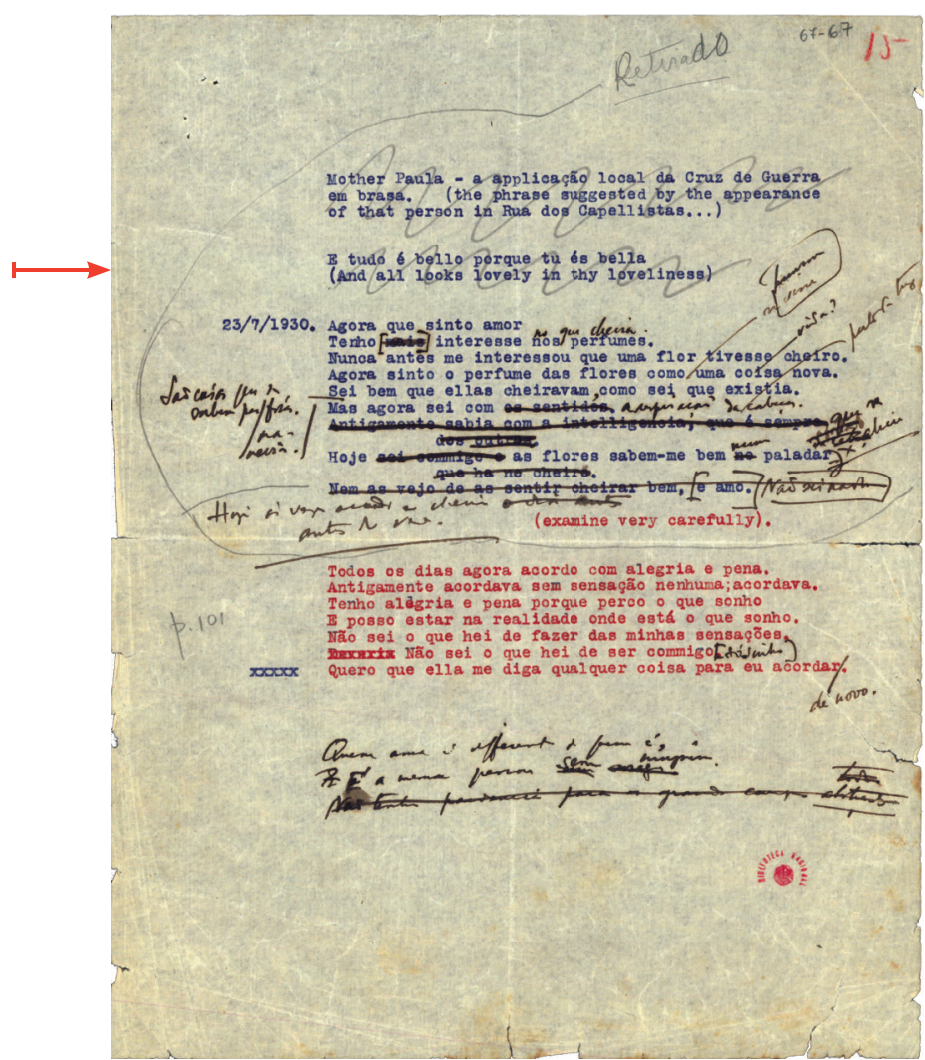

Fig. 1 : Fernando Pessoa,

Bibliothèque nationale du Portugal, Esp. E 3, 67-67r

nous a permis d'identifier plusieurs fonctions de l'usage des langues étrangères dans le processus créatif, notamment : (1) l'emprunt et la citation, (2) les annotations métadiscursives, et (3) le changement de langue comme moteur de l'écriture.

\section{Emprunts et citations en une autre langue}

En ce qui concerne les emprunts et les citations que l'on peut trouver dans les documents d'archives, on ne constate pas de différence fondamentale par rapport aux textes publiés du vivant de Pessoa. Chez lui, on observe notamment la présence - somme toute assez répandue chez des écrivains plurilingues - d'épigraphes écrites dans une langue qui diffère de celle utilisée pour la création du texte 5 .

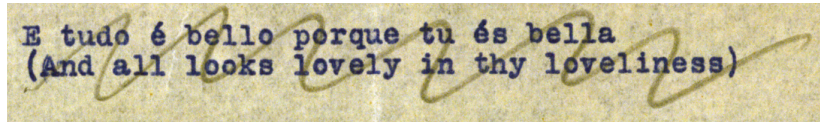

Fig. 2 : Fernando Pessoa,

Bibliothèque nationale du Portugal, Esp. E 3, 67-67r (détail)

Par exemple, dans le document 67-67 r (fig. 1) contenant plusieurs segments dactylographiés (dont le dernier est un poème attribué à l' hétéronyme Alberto Caeiro $^{6}$ ) et quelques corrections et ajouts manuscrits, on voit une inscription en portugais doublée de ce qui semble être sa traduction en anglais : «E tudo é bello porque tu és bella /(And all looks lovely in thy loveliness)» (fig. 2).

On pourrait croire que Pessoa est l'auteur de ces deux segments. Or, comme le signalent Fernando Cabral Martins et Richard Zenith, le segment anglais «And all looks lovely in thy loveliness » n'est pas de Pessoa, mais appartient au sonnet «Love's blindness » du poète victorien tardif Alfred Austin (1835-1913)7 . Cette identification nous met en garde quant à l'attribution des passages mis entre parenthèses par l'écrivain.

Dès lors, il s'agit ici, certes, d'écriture plurilingue, mais plus exactement d'une citation en anglais traduite

5. Par exemple, «Nescio quid meditans» (Poemas de Fernando Pessoa1921-1930, éd. Ivo Castro, Lisboa, Imprensa Nacional - Casa da Moeda, 2001, p. 220), «Un Soir à Lima» (Poemas de Fernando Pessoa 1934-1935, éd. Luís Prista, Lisboa, Imprensa Nacional - Casa da Moeda, 2000, p. 232-241), «Procrastinators, banditti» (Poemas de Fernando Pessoa. Poemas Ingleses, tomo II, éd. João Dionísio, Lisboa, Imprensa Nacional - Casa da Moeda, 1997, p. 312).

6. On compte d'habitude plus de 100 figures hétéronymiques créées par Fernando Pessoa. À ce sujet, voir Fernando Cabral Martins (dir.), Dicionário de Fernando Pessoa e do Modernismo Português, Lisboa, Caminho, 2008 (notamment, Fernando Cabral Martins, «Heteronímia História», p. 326-327; Fernando Guimarães, «Heteronímia - Poética», p. 327-333; Fernando Cabral Martins, «Heterónimo», p. 333-334); Fernando Pessoa, Teoria da heteronímia, éd. Fernando Cabral Martins et Richard Zenith, Lisboa, Assírio \& Alvim, 2012; Jerónimo Pizarro et Patricio Ferrari (dir.), Fernando Pessoa, Eu Sou uma Antologia. 136 Autores Fictícios, Lisboa, Tinta da China, 2013; Pedro Sepúlveda, Os livros de Fernando Pessoa, Lisboa, Ática, 2013.

7. Alberto Caeiro, Poesia, éd. Fernando Cabral Martins et Richard Zenith, Lisboa, Assírio \& Alvim, 2001, p. 214. Sur l'opinion de Pessoa à propos de ce poème d'Austin, voir Carlos Pittella, «Sonnet 101 with Prof. Pessoa. Fernando Pessoa's Marginalia on an Anthology of 19th-Century English Sonnets », Pessoa Plural 11, P./Spr. 2017, p. 336. 
en portugais et dépourvue de traitement ultérieur. La mise entre parenthèses renverse le statut de la citation en langue étrangère, en lui conférant une place secondaire ou en étant laissée uniquement à titre de rappel (comme une sorte de procédé mnémotechnique).

\section{Annotations métadiscursives}

À première vue, un des aspects les plus frappants dans les archives de Pessoa est peut-être la présence de notes de régie, rédigées fréquemment en anglais, que l'auteur semble employer afin de s'orienter dans la rédaction et dans la relecture de son propre texte. Par exemple, Pessoa a recours à des indications métadiscursives comme over (l'équivalent de l'instruction «tournez la page » ou «de l'autre côté de la feuille») qui lui permettent de s'orienter pendant la rédaction du texte; ou bien to (suivi de l'indication du numéro de la page ou de la feuille vers où le regard doit se diriger). L'écrivain se sert également d'indications en anglais quand il se réfère au statut de textes manuscrits, comme copied, signifiant que ce texte a été retranscrit dans un autre document. Enfin, certains textes reçoivent des appréciations, également en anglais, aussi bien que des indications sur le chemin à suivre dans la construction textuelle. Ces données font penser que l'anglais a une fonction d'outil métadiscursif qui instaure une distance par rapport à la langue de rédaction.

Ce rôle rempli par l'anglais est particulièrement manifeste dans les plans. Une liste d'incipit de poèmes écrits en portugais attribués à l'hétéronyme Alberto Caeiro (fig. 3), rédigée autour de mars 1914 et qui s'arrête au numéro 45 (bien que ni le numéro 44 ni le 45 ne soient associés à un incipit), fait l'objet du commentaire suivant: (perhaps there are more than these). I (Try to reach 50, or, at the very least, 45) I or $49(44,45,46,47,48,49)^{8}$.

Dans un autre brouillon de poème attribué également à Alberto Caeiro 9 (fig. 4), on voit une instruction en anglais ( $a d d$ something here), ajoutée à la fin du poème, également entre parenthèses, instruction qui ne sera d'ailleurs pas suivie.

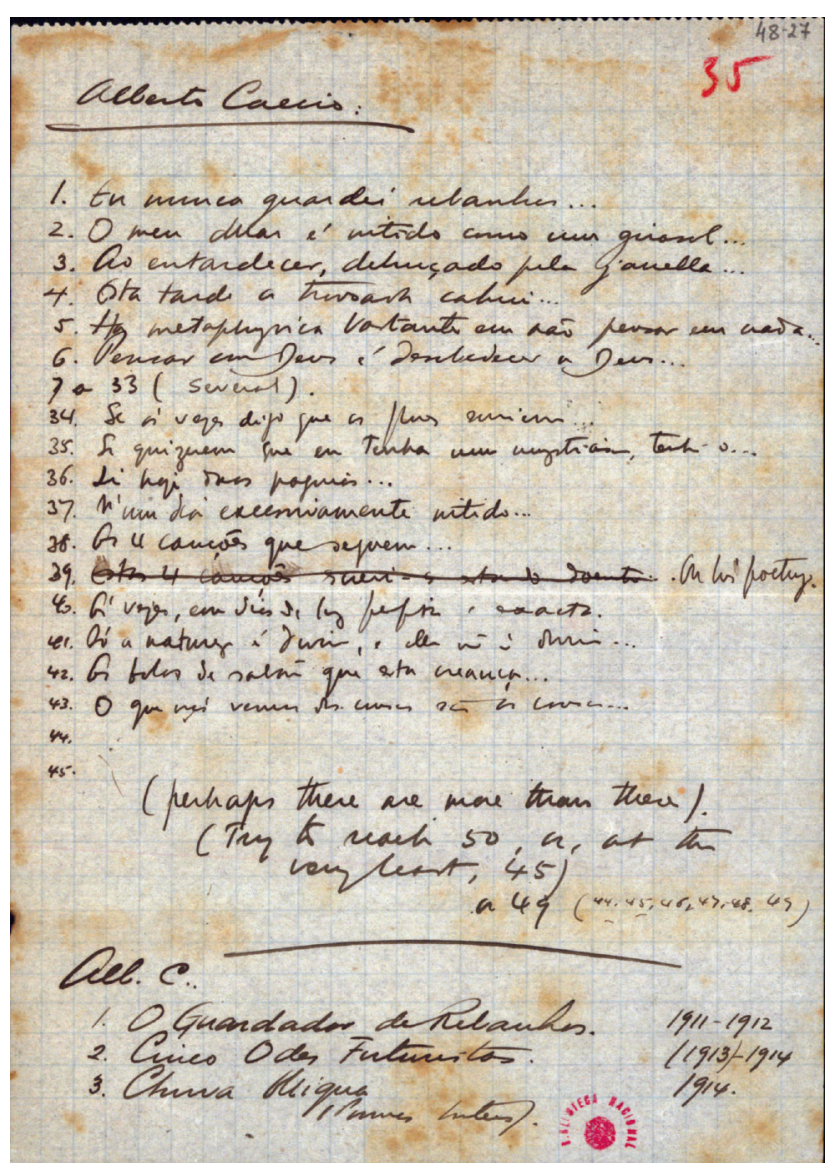

Fig. 3 : Fernando Pessoa, Bibliothèque nationale du Portugal, Esp. E 3, 48-27r, Liste de poèmes attribués à Alberto Caeiro

Avec un rôle moins performatif (et un statut génétique moins transparent) que dans les deux cas déjà mentionnés, l'anglais sert également à introduire des commentaires explicatifs dans un plan (fig. 5) ${ }^{10}$. La dernière entrée de ce plan porte un titre provisoire «(Os Emigrantes)», qui est

8. «(Peut-être y en a-t-il plus que cela). (Essayer d'atteindre 50, ou, au strict minimum, 45) ou $49(44,45,46,47,48,49) »$ : Pedro Sepúlveda et Jorge Uribe, O planeamento editorial de Fernando Pessoa, Lisboa, Imprensa Nacional - Casa da Moeda, 2016, p. 63-64.

9. Fernando Pessoa, Poemas de Alberto Caeiro, éd. Ivo Castro, Lisboa, Imprensa Nacional - Casa da Moeda, 2015, p. 170-171.

10. Ce plan a été publié dans Fernando Pessoa, O Marinheiro, éd. Cláudia Franco Souza, Lisboa, Ática, 2010, p. 10. 


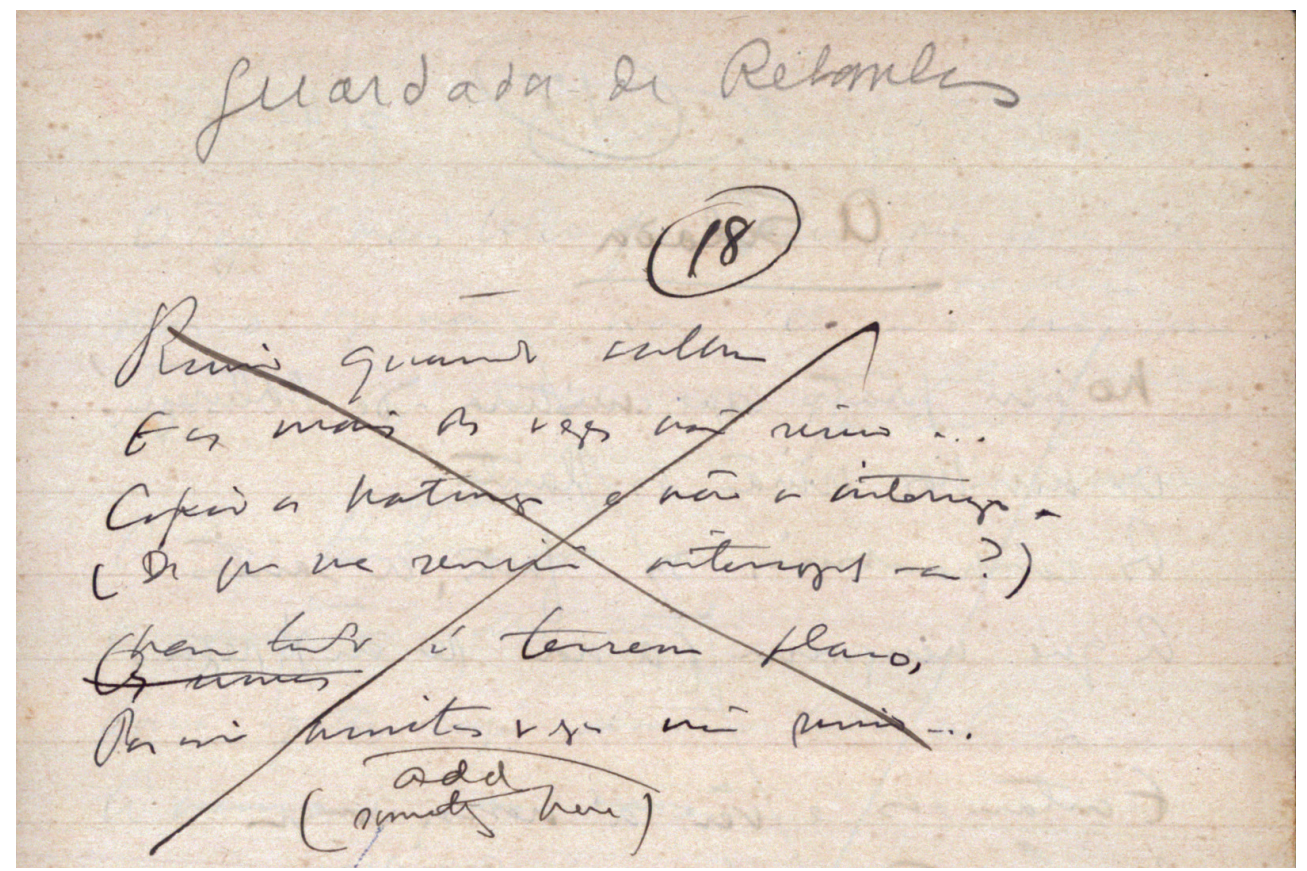

Rimo quando calha

E as mais das vezes não rimo...

Copio a Natureza e não a interrogo.

(De que me serviria interrogal-a?)

As rimasNem tudo é terreno plano,

Por isso muitas vezes não rimo... (add I something here)
Je rime quand ça tombe bien

Et le plus souvent je ne rime pas...

Je copie la nature, je ne l'interroge pas.

(À quoi m'aurait servi de l'interroger?)

Les rimesTout n'est pas terrain plat,

C'est pour ça que bien souvent je ne rime pas

(ajouter I quelque chose ici)

Fig. 4 : Fernando Pessoa, Bibliothèque nationale du Portugal, Esp. E 3, 67-29v (reproduction partiale), « Rimo quando calha »

commenté en anglais : «children who pretend to emigrate, and their ardour of otherness $11_{\Perp}$.

Dans ces trois cas, l'anglais sert d'outil métadiscursif au poète, en illustrant par cet usage la stratégie créative de distinction fonctionnelle des langues ${ }^{12}$ qui consiste en l'utilisation d'une langue pour des fonctions extratextuelles, alors que l'autre système linguistique fournit la matière verbale du texte.

On peut ajouter ici que la distinction fonctionnelle des langues s'observe aussi dans la bibliothèque particulière de Fernando Pessoa ${ }^{13}$, puisque la fonction de commentaire en marge de plusieurs ouvrages est quelquefois assignée à une langue différente du système linguistique de l'ouvrage. Voyons, par exemple, la page 3 de l'ouvrage d'Alfred Fouillée, La Philosophie de Platon: théorie des idées et de l'amour ${ }^{14}$, dont la marge supérieure est occupée par l'annotation «Reasoning needs principles, and these are ideas; but reasoning cannot be used to prove the existence of its own principles for it supposes them» [Le raisonnement requiert des principes et ceux-ci sont des idées; mais le raisonnement ne peut pas être utilisé pour prouver l'existence de ses propres principes car il les suppose] (fig. 6).

Mais, dans d'autres cas, ce rôle est joué par la langue portugaise, comme c'est le cas des qualificatifs estupendo

11. Pour une glose explicative similaire, voir Fernando Pessoa, Livro do Desasocego, éd. Jerónimo Pizarro, I, Lisboa, Imprensa Nacional - Casa da Moeda, 2010, p. 83.

12. Voir le détail de la typologie des stratégies créatives dans Olga Anokhina, «Étudier les écrivains plurilingues grâce aux manuscrits », dans Olga Anokhina et François Rastier (dir.), Écrire en langues : littératures et plurilinguisme, Paris, Éditions des archives contemporaines, coll. «Multilinguisme, traduction, création», 2015, p. 31-43.

13. Jerónimo Pizarro, Patricio Ferrari et Antonio Cardiello, A Biblioteca particular de Fernando Pessoa, vol. I, Lisboa, Dom Quixote, 2010, p. 78-79.

14. Alfred Fouillée, La Philosophie de Platon : théorie des idées et de l'amour, 3e éd., Paris, Librairie Hachette et Cie., $1904<$ http://casafernandopessoa.cm-lisboa.pt/bdigital/1-49> (consulté en décembre 2017) et Jerónimo Pizarro, Patricio Ferrari et Antonio Cardiello, op. cit., p. 78-79. 


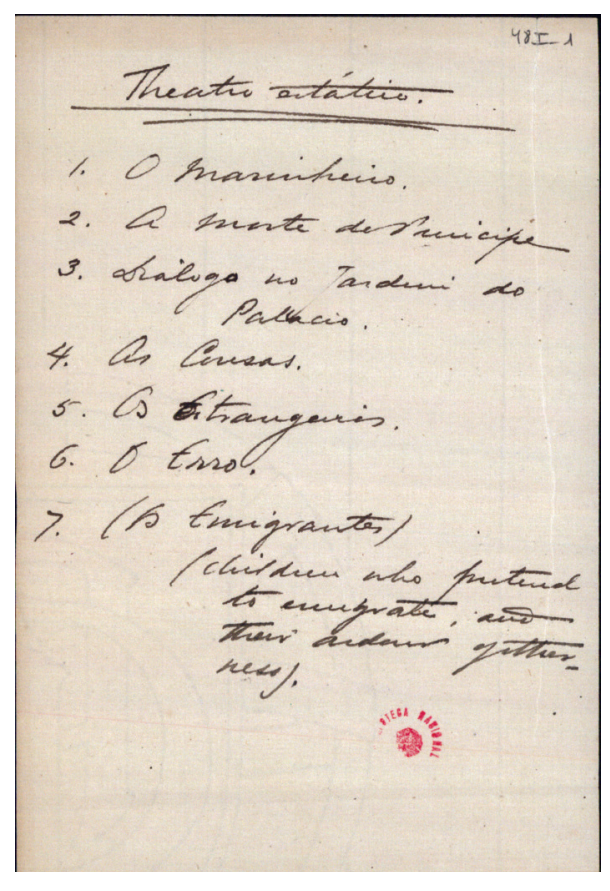

Theatro estático.

1. O Marinheiro.

2. A morte do Principe

3. Dialogo no Jardim do Palacio.

4. As Cousas.

5. Os Estrangeiros.

6. O Erro.

7. (Os Emigrantes) (children who pretend to emigrate, and their ardour of otherness).
Théâtre statique.

Le Marin.

La mort du Prince

Le dialogue dans le jardin du

Palais.

Les Choses.

Les Étrangers.

L'Erreur.

(Les Émigrants)

(les enfants qui font semblant

d'émigrer et

leur ardeur d'altérité.

Fig. 5 : Fernando Pessoa, Bibliothèque nationale du Portugal,

Esp. E 3, 48I-1r, liste de textes dramatiques

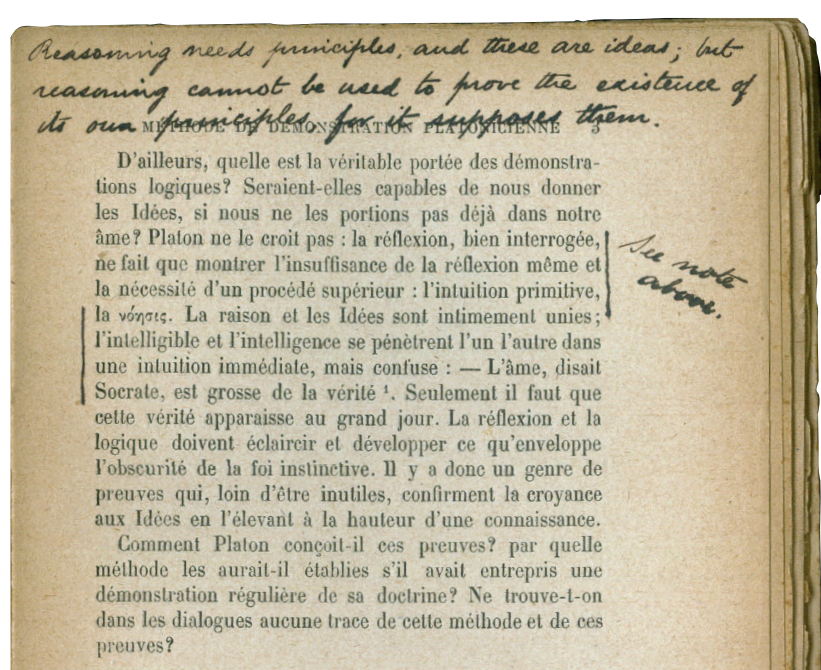

Fig. 6 : Casa Fernando Pessoa, l'ouvrage de la bibliothèque personnelle de l'écrivain: Alfred Fouillée, La Philosophie de Platon: théorie des idées et de l'amour, p. 3 (détail)

[extraordinaire] et justissimo [très juste], qu'on trouve en marge de la page 9 d'Henri-Frédéric Amiel, Fragments d'un journal intime, précédés d'une étude par Edmond Scherer 15 (fig. 7). Cependant, il faut déterminer si cette annotation marginale tout comme toutes les autres est bien de la main de l'écrivain.

La situation est similaire en ce qui concerne les textes et notes dits «automatiques », où la prédominance nette de l'anglais n'efface pas la présence occasionnelle du portugais. Par conséquent, bien que Pessoa utilise fréquemment

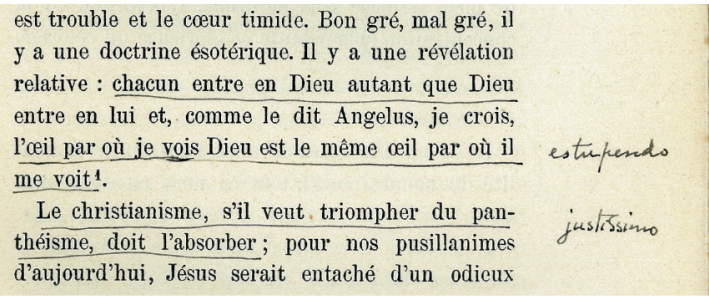

Fig. 7 : Casa Fernando Pessoa, l'ouvrage de la bibliothèque personnelle de l'écrivain : Henri-Frédéric Amiel, Fragments d'un journal intime, précédés d'une étude par Edmond Scherer, p. 9 (détail)

l'anglais comme «langue mentale», le portugais peut aussi jouer ce rôle16.

\section{Changement de langue comme moteur de l'écriture}

On peut observer un troisième type d'usage des langues étrangères chez Pessoa, dans le dossier génétique du «drame

15. Henri-Frédéric Amiel, Fragments d'un journal intime, précédés d'une étude par Edmond Scherer, 11e édition, tome I, Genève, Georg et $\mathrm{Co}^{\mathrm{e}}$ libraires-éditeurs, $1911<$ http://casafernandopessoa.cm-lisboa.pt/ bdigital/8-7/2> (consulté en décembre 2017).

16. Cf. Fernando Pessoa, Escritos autobiográficos, automáticos e de reflexão pessoal, éd. Richard Zenith, Lisboa, Assírio \& Alvim, 2003 ; et Poemas de Fernando Pessoa - 1915-1920, éd. João Dionísio, Lisboa, Imprensa Nacional-Casa da Moeda, 2005, p. 103, poème 106. 
statique $O$ Marinheiro ${ }^{17}$ [Le Marin], étudié en détail par Claudia Fischer ${ }^{18}$ : celui-ci est fondamentalement différent du cas de la citation que nous avons évoqué plus haut.

Bien que les documents écrits en français liés au poème dramatique $O$ Marinheiro soient souvent interprétés comme étant une traduction réalisée par Pessoa à partir du portugais, Fischer argumente de manière convaincante qu'une autre vision de ce texte est possible, défendant deux hypothèses : 1) contrairement à l'opinion répandue, c'est la version française qui précède le texte portugais et, par conséquent, l'autotraduction s'opère non pas du portugais vers le français mais dans le sens inverse; 2) par ailleurs, il ne s'agit pas exactement d'une autotraduction (dans le sens d'une version hiérarchiquement subordonnée à un texte antérieur), mais d'une genèse bilingue ${ }^{19}$. En effet, Claudia Fischer affirme que, non seulement le texte français est bien premier mais que, par moments, Pessoa convoque au fil de l'écriture la langue portugaise. D'où les deux hypothèses initiales de Fischer : la primauté du français et la co-présence des deux langues dans la genèse de l'œuvre.

L'analyse des documents d'archives qui ont un rapport avec $O$ Marinheiro suggère que Pessoa, agissant comme Oscar Wilde quand il composait Salome (écrit originalement en français), a voulu - lui aussi - situer son drame dans le sillage de ses lectures de Maeterlinck ${ }^{20}$. Bien qu'il ne soit pas nécessaire de reprendre dans son intégralité toute l'interprétation de Fischer, il s'avère utile de présenter ici deux documents du dossier génétique de $O$ Marinheiro qui la soutiennent. Le premier est le manuscrit 74B-20 (fig. 8), qui témoigne du changement de code linguistique au moment où le poète semble rencontrer une difficulté pour trouver le mot juste en français 21 :

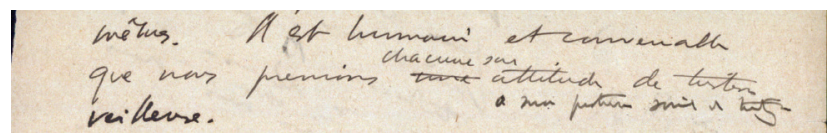

Fig. 8 : Fernando Pessoa, Bibliothèque nationale du Portugal, Esp. 3, 74B-20r (détail), version en français et portugais de $O$ Marinheiro

(...). Il est humain et convenable

$$
\text { chacune son }
$$

que nous prenions une attitude de tristesse

a sua postura servil de tristeza

veilleuse.
Visiblement, Pessoa hésite au moment où il écrit le complément d'objet direct de «nous prenions» : «une attitude de tristesse». Il élimine ensuite l'article indéfini «une» en le remplaçant par «chacune son» dans l'espace interlinéaire supérieur. Puis, insatisfait du résultat, il change de code linguistique et écrit en portugais dans l'interligne inférieur «a sua postura servil de tristeza» [sa posture servile de tristesse]. Cet exemple documente de manière flagrante l'instant où un code linguistique contraignant (en l'occurrence le français) cède sa place à un autre code linguistique qui offre au poète davantage de liberté et de sécurité (ici, le portugais).

En analysant un autre document, 74B-22 (fig. 9), un passage plus développé permet de mettre en lumière comment s'opère la transition de l'écriture en français vers l'écriture en portugais (fig. 9).

Dans la figure 8 et dans la partie supérieure de la figure 9, l'intervention en portugais se matérialise comme une révision immédiate qui se situe en interligne par rapport au texte français. On remarquera que cette intervention ne semble pas correspondre à une campagne de révision ultérieure, mais qu'elle appartient à la même phase que l'écriture en français. En effet, le caractère immédiat du changement de code linguistique dans ces interventions est suggéré par le fait que Pessoa ne change pas d'encre quand il passe du français au portugais. Ceci permet de souligner une particularité du document 9 : entre la réplique qui commence par «Peut-être» et la réplique suivante, commençant avec l'appel «Não falleis mais » [Ne parlez plus],

17. Publié dans la revue iconique du premier modernisme portugais, Orpheu I, 1915. En ligne : < http://purl.pt/12089> (consulté en décembre 2017). Cf. la traduction française, Le marin : drame statique en un tableau, préf. José Augusto Seabra, trad. Bernard Sésé, Paris, José Corti, 1999.

18. Claudia J. Fischer, «Auto-tradução e experimentação linguística na génese d" "O Marinheiro" de Fernando Pessoa», Pessoa Plural I (P./Spr. 2012), p. 1-69 < http://www.brown.edu/Departments/Portuguese_ Brazilian_Studies/ejph/pessoaplural/Issue1/PDF/I1A01.pdf > (consulté en décembre 2017).

19. Claudia J. Fischer, op. cit., p. 13.

20. Claudia J. Fischer, op. cit., p. 16.

21. On reproduit diplomatiquement la transcription de Fischer. Ici et plus loin, les segments écrits en portugais sont transcrits en italique. 


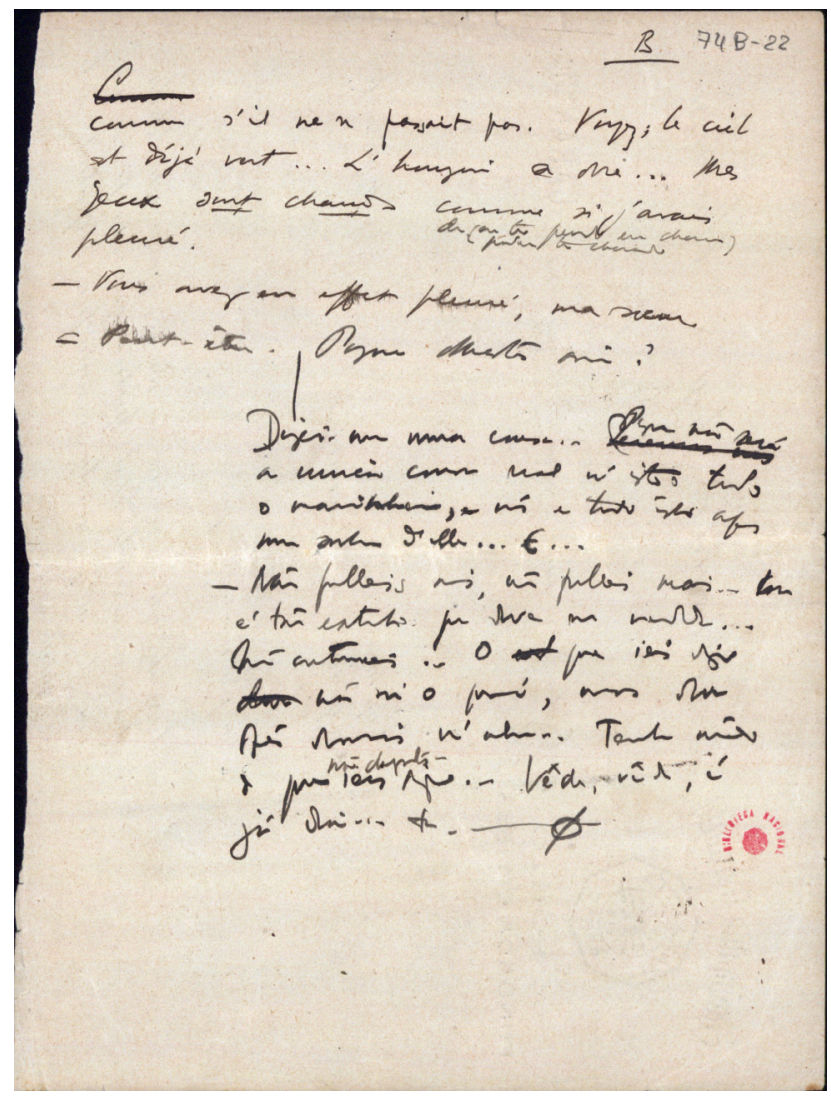

Comme

comme s'il ne se passait pas. Voyez; le ciel est déjà vert... L'horizon se dore... Mes yeux sont chauds comme si j'avais de (eu ter pensado em chorar)

pleuré. poder ter chorado *

- Vous avez en effet pleuré, ma soeur.

- Peut-être. Porque olhastes assim?

Porque não será
Dizei-me uma cousa...
a unica cousa real n'isto tudo
o marinheiro, e nós e tudo isto apenas
um sonho d'elle... E... **
- Não falleis mais, não falleis mais... Isso
é tão estranho que deve ser verdade...
Não continueis... O [mot illisibłeł que ieis dizer
teve não sei o que é, mas deve
sêr demais n'alma... Tenho mêdo
não chegastes a
do que ieis dizer.-Vêde, vêde, é
já dia... etc. - ${ }^{* * *}$.

Fig. 9 : Fernando Pessoa, Bibliothèque nationale du Portugal, Esp. 3, 74B-22r, version en français et portugais de $O$ Marinheiro

(*) Traduction du segment portugais : «de (avoir pensé pleurer) [ $\downarrow$ pouvoir avoir pleuré]».

(**) Traduction du segment portugais : «Pourquoi avez-vous regardé comme ça ? $\downarrow$ Dites-moi une chose... Serions-nous [ $\uparrow$ Pourquoi ne serait-ce pas] le matelot l'unique chose réelle en tout cela, et nous et tout cela seulement son rêve... Et...».

(***) Traduction du segment portugais : «Ne parlez plus, ne parlez plus... Ceci est si étrange que ce doit être vrai... Ne continuez pas... Ce mot illisible que vous étiez en train de dire doit je ne sais pas ce que c'est, mais ce doit être trop dans l'âme... J'ai peur de ce que vous alliez [ $\uparrow$ vous n'êtes pas arrivés à] dire. - Voyez, voyez, il fait déjà jour... etc. ».

le portugais cesse d'être la langue de rédaction alternative pour devenir la langue principale.

L'analyse du dossier génétique de la pièce Marinheiro auquel appartiennent ces deux documents amène Fischer à intégrer Pessoa dans le groupe de ces auteurs bilingues qui s'engagent dans un processus de création littéraire et qui, après s'être lancés dans une écriture, passent à un autre système linguistique 22 .

C'est également le cas du poème suivant (fig. 10) qui commence par un tercet en français et qui dérive très rapidement vers l'écriture en langue portugaise ${ }^{23}$.

Après avoir écrit les trois premiers vers en français, Pessoa les élimine et recommence en portugais : l'incipit est égal au début du tercet, le deuxième vers possède une structure semblable au vers correspondant en français et on assiste ensuite à un changement de direction, avec la définition de la forme strophique (le quatrain) et de la longueur du poème (12 vers). D'une manière différente de ce qu' on a pu voir à propos du «Marin», le passage du français au portugais est ici moins progressif et se manifeste par l'existence de deux blocs textuels. Toutefois, comme dans le cas du «Marin», l'écriture en français stimule la création poétique en portugais. Le changement de système linguistique est sans doute motivé par la plus grande compétence de l'écrivain en portugais. Ajoutons qu'on rencontre dans quelques-uns des essais critiques de Pessoa des cas très similaires de

22. Claudia J. Fischer, op. cit., p. 21.

23. Poemas de Fernando Pessoa - 1934-1935, éd. Luís Prista, Lisboa: Imprensa Nacional - Casa da Moeda, 2000, p. 144 et 384 (nous reproduisons le texte selon cette édition, mais diplomatiquement). Sur ce poème, voir Patricio Ferrari, «Pour une édition critique des poèmes français de Fernando Pessoa et d'Alejandra Pizarnik », dans Bilinguisme, double culture, littératures, Paris, L'Harmattan, 2012, p. 441-442. Voir aussi Fernando Pessoa, Euvres poétiques, préf. Robert Bréchon, éd. Patrick Quillier, Paris, Gallimard, 2001, p. 1617 et 2047. 

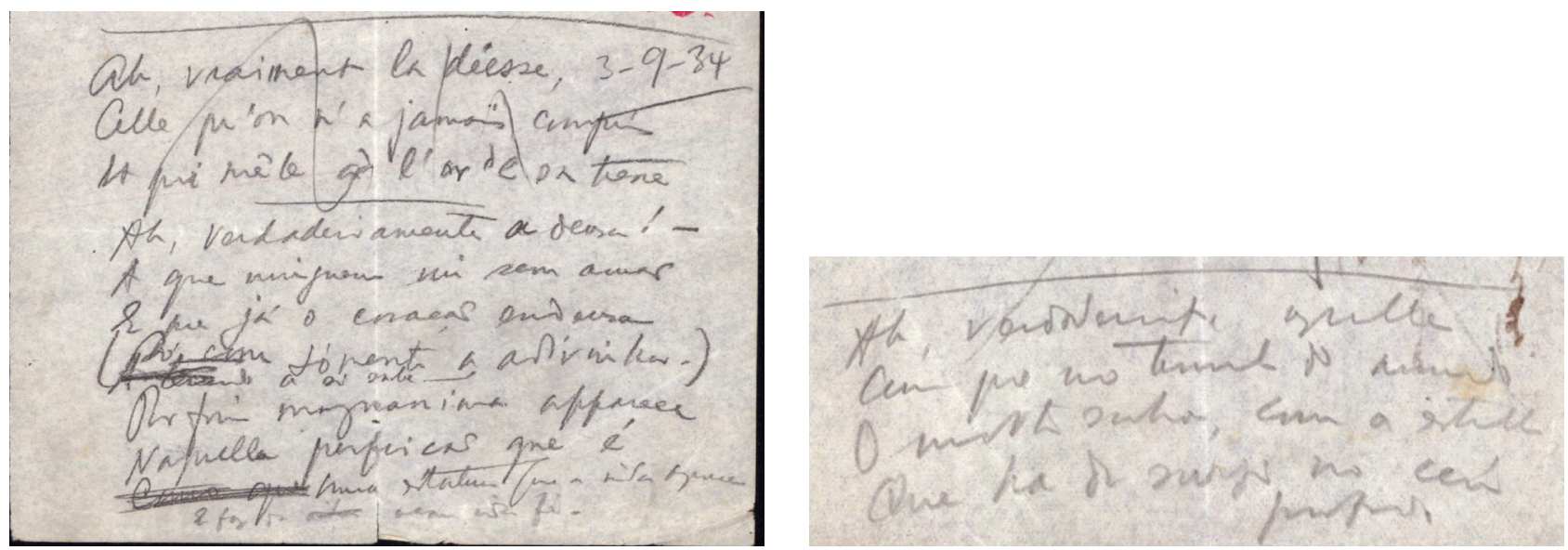

Fig. 10 : Fernando Pessoa, Bibliothèque nationale du Portugal, Esp. 3, 33-42r et 42v (détails) : «Ah, verdadeiramente a deusa !»-, le tercet en français et ensuite les deux premières strophes du poème portugais (33-42r), la troisième strophe en portugais $(33-42 \mathrm{v})$

Ah, vraiment la déesse,

Celle qu'on n'a jamais comprise

Et qui mêle à l'or de sa tresse

Ah, verdadeiramente a deusa! -

A que ninguem viu sem amar

E que já o coração endeusa

Só com

Antes sómente a adivinhar.

Quando a só sabe

Por fim magnanima apparece

Naquella perfeição que é

Como que Uma estatua que a vida aquece

$\mathrm{E}$ faz da vida mesma vida fé.

Ah, verdadeiramente aquella

Com que no tumulo do mundo

O morto sonha, como a estrella

Que ha de surgir no céu profundo.
[Traduction du texte portugais :

Ah, vraiment la déesse,

Que personne n'a vue sans l'aimer

Et que déjà le cœur déifie

Seule avec

Avant seulement la deviner

Quand seule la sait

Enfin magnanime apparaît

En cette perfection qui est

Comme que Une statue que la vie chauffe

Et fait de la vie vie elle-même foi.

Ah, vraiment celle

À qui dans le tumulte du monde

Le mort songe, comme l'étoile

Qui va surgir au ciel profond.] 
changement de code, quand l'écriture en anglais cède la place à l'écriture en langue portugaise 24 .

\section{Conclusion}

Le plurilinguisme littéraire visible dans les documents de travail des écrivains peut être objet de plusieurs approches critiques qui se développent en conformité avec les archives étudiées : dans cet article, nous nous sommes bornés à attirer l'attention sur quelques manifestations de recours à d'autres langues que le portugais chez Fernando Pessoa. Étant donné que l'usage de ces langues peut viser plusieurs fins, nous nous sommes concentrés, outre le cas fréquent de la citation, sur deux types de phénomènes bien singuliers.

Il s'agit en premier lieu des annotations métadiscursives, y compris des instructions d'élaboration poétique, qui apparaissent fréquemment en anglais. En second lieu, les manifestations peut-être les plus intéressantes du bilinguisme à l'œuvre sont motivées par une «perte d'haleine 25 », quand, ayant commencé un texte dans une langue, Pessoa décide de changer de code linguistique et continue ou reprend l'écriture en portugais dans lequel il se sent beaucoup plus à l'aise. À cet égard, le français fonctionne occasionnellement comme langue d'inspiration littéraire.

Ce type de plurilinguisme manifeste dans le processus créatif peut être mis en relation avec la vision idéaliste que Pessoa a de la poésie, qui «tem que ser composta e expressa numa língua qualquer» [doit être composée et exprimée dans une langue quelconque] ${ }^{26}$. L'auteur est forcé de choisir une langue parce que, conformément à ce qu'il déclare quand il se prononce sur la viabilité d'un langage universel, «there is no language of that sort except it be of the spirit» [il n'y a pas de langage de ce genre, sauf celui de l'esprit]27.

Autrement dit, pour qu'une langue donnée ressemble un peu à ce langage de l'esprit, Pessoa affirme que :

[...] the language be as pliant as possible, so that there be in it as full a capacity for expression of all moods as can be, a consequent capacity to admit, by translation, the reflex of other languages and thus dispense, from the literary standpoint, with the learning of them.

[(...) le langage doit être aussi flexible que possible, afin qu'il y ait en lui une capacité d'expression de toutes les disposi- tions aussi grande que possible, une capacité conséquente à admettre, à travers la traduction, le reflet d'autres langues et ainsi de dispenser, du point de vue littéraire, de la nécessité de les apprendre ${ }^{28}$.

Ainsi, la langue utilisée par un poète doit être aussi flexible que possible : dans un autre texte, Pessoa parlera du portugais comme «la langue la plus flexible et complexe des langues romanes ${ }^{29}$. Cette ductilité s'allie à la disposition des règles grammaticales de la langue naturelle à être modifiées en conformité avec ce que l'on veut exprimer. Dès lors, écrit Pessoa,

é legítimo violar as mais elementares regras da gramática - no estilo expositivo ou no artístico - se com isso ou a ideia ganha clareza ou firmeza, ou à frase se enriquece o seu conteúdo de sugestão.

[il est légitime de violer les règles de grammaire les plus élémentaires, dans le style expositif ou artistique, si avec ceci l'idée gagne en clarté ou fermeté, ou si le contenu de suggestion de la phrase s'enrichit.]

La recherche de la clarté ou de la fermeté verbale, qui constitue un axe principal de l'expérience littéraire de Pessoa, est éclairée par ses expériences de bilinguisme créatif, notamment celles où l'observateur le surprend à des moments de mise en marche et de réévaluation de la langue qu'il utilise.

24. Voir Fernando Pessoa, A Língua Portuguesa, éd. Luísa Medeiros, Lisboa, Assírio \& Alvim, 1997, p. 150 ; (cf. Esp. 3, 123-96r) et Rita Patrício, Episódios. Da teorização estética em Fernando Pessoa, Vila Nova de Famalicão, Húmus - Centro de Estudos Humanísticos da Universidade do Minho, 2012, p. 375-376 (cf. Esp. 3, 18-4).

25. Nous empruntons cette expression à Patricio Ferrari, «Pour une édition critique des poèmes français de Fernando Pessoa et d'Alejandra Pizarnik », dans Cristina Pirvu (dir.), Bilinguisme, double culture, littératures, Paris, L'Harmattan, 2012, p. 440

26. Rita Patrício, op. cit., p. 83 et p. 325-326.

27. Fernando Pessoa, A Língua Portuguesa, éd. Luísa Medeiros, Lisboa, Assírio \& Alvim, 1997, p. 96. Curieusement, on trouve des textes bilingues parmi les écrits dits automatiques de Pessoa (voir Fernando Pessoa, Escritos autobiográficos, automáticos e de reflexão pessoal, éd. Richard Zenith, collab. Manuela Parreira da Silva, trad. Manuela Rocha, Lisboa, Assírio \& Alvim, 2003, p. 282 et 326).

28. Fernando Pessoa, op. cit., p. 147.

29. Rita Patrício, op. cit., p. 305. 
João Dionísıo est professeur de critique textuelle et spécialiste de Fernando Pessoa à la faculté de Lettres de l'université de Lisbonne, chercheur au Centre de linguistique de la même université. Il a présidé la European Society for Textual Scholarship (2013-2016). Membre de l'équipe Pessoa depuis sa fondation, il est responsable de trois volumes de l'édition critique génétique de l'œuvre de Fernando Pessoa.

joaodionisio@campus.ul.pt

\section{Remarques sur la création plurilingue chez Fernando Pessoa}

L'observation du fonds Fernando Pessoa (1888-1935) à la Bibliothèque nationale du Portugal permet de mettre en évidence deux types de recours à d'autres systèmes linguistiques que la langue maternelle de l'écrivain portugais (1888-1935) : 1) annotations métadiscursives, inclusivement des indications de création littéraire, dont un nombre significatif en anglais ; 2) l'abandon de la langue dans laquelle il commence à écrire un texte (l'anglais ou le français, langues occasionnelles d'inspiration initiale) pour continuer en portugais. Ce type de bilinguisme manifeste dans le processus créatif est mis en rapport avec la vision idéaliste que Pessoa a de la poésie, qui, selon lui, «doit être composée et exprimée dans une langue quelconque», comme si toute création littéraire était le résultat de la traduction du langage de l'esprit vers une langue naturelle.

The study of the Fernando Pessoa collection at Portugal's National Library reveals that this Portuguese writer (1888-1935) resorted to linguistic systems other than his maternal language in two different ways: 1) metadiscursive annotations, including indications of literary creation, many in English; 2) his dropping of the language used at the start (English or French, occasional languages of the initial inspiration), to continue in Portuguese. This type of bilingualism evident in the creative process is considered in relation to the idealistic vision Pessoa has of poetry, which, in his view, "should be composed and expressed in any language", as if all literary creation were the result of a translation from a spiritual language to a maternal one.

Beim Einblick in den Nachlass von Fernando Pessoa in der Nationalbibliothek von Portugal zeigt sich, dass der portugiesische Schriftsteller (1888-1935) auf zweierlei Art auf andere sprachliche Systeme als auf die Muttersprache zurückgreift: 1) metadiskursive Annotationen, einschließlich Hinweise auf literarisches Schaffen, davon eine bedeutende Anzahl auf Englisch; 2) das Hintersichlassen jener Sprache, in der er beginnt, einen Text zu schreiben (Englisch oder Französisch: Sprachen, die er gelegentlich zur anfänglichen Inspiration verwendet), um auf Portugiesisch fortzufahren. Diese Art von Zweisprachigkeit, die sich im schöpferischen Prozess manifestiert, hängt mit der idealistischen Vision zusammen, die Pessoa für die Poesie hat, die, wie er sagt, ,,in irgendeiner Sprache konzipiert und ausgedrückt werden muss", als ob jede literarische Schöpfung das Ergebnis einer Übersetzung aus einer Ideensprache in eine natürliche Sprache sei.
La observación del Fondo Fernando Pessoa en la Biblioteca Nacional de Portugal permite poner en evidencia dos tipos de usos de sistemas lingüísticos diferentes de la lengua materna de este escritor portugués (1888-1935): 1) anotaciones metadiscursivas, entre las cuales indicaciones de creación literaria, por una gran parte en inglés; 2) abandono del idioma en el que comienza a escribir un texto (inglés o francés, lenguas ocasionales de inspiración inicial) para proseguir en portugués. Este tipo de bilingüismo que se pone de manifiesto en el proceso creativo será vinculado con la visión idealista que Pessoa tiene de la poesía, la cual, según él, "debe ser compuesta y expresada en la lengua que sea", como si toda creación literaria fuera el resultado de una traducción de una lengua espiritual a una lengua natural.

A observação do espólio de Fernando Pessoa na Biblioteca Nacional de Portugal permite destacar dois tipos de recurso a sistemas linguísticos diferentes do da sua língua materna na prática literária deste escritor português (1888-1935): 1) anotações metadiscursivas, incluindo indicações de criação literária, que aparecem em número significativo em inglês; 2) o abandono da língua com que tinha começado a escrever (inglês ou francês, idiomas ocasionais de inspiração inicial) para de seguida continuar em português. Este tipo de bilinguismo expresso no processo de criação é relacionado com a visão idealista que Pessoa defendia da poesia, que, segundo, ele, «tem que ser composta e expressa numa língua qualquer», como se toda a criação literária fosse o resultado da tradução de uma língua espiritual numa língua natural.

L'analisi del fondo Ferdinando Pessoa della Biblioteca nazionale del Portogallo permette di evidenziare, nella pratica letteraria dello scrittore portoghese (1888-1935), due tipi di ricorso ad altri sistemi linguistici diversi dalla lingua materna: 1) annotazioni meta-discorsive, comprese alcune indicazioni di creazione letteraria, di cui un numero significativo in inglese; 2) l'abbandono della lingua in cui inizia a scrivere un testo (l'inglese o il francese, lingue occasionali d'ispirazione iniziale) per continuare in portoghese. Questo tipo di bilinguismo che si manifesta nel processo creativo è messo in relazione con la visione idealista della poesia che, secondo Pessoa, "deve essere composta ed espressa in una lingua qualsiasi”, come se qualsiasi creazione letteraria fosse il risultato della traduzione da una lingua spirituale a una lingua naturale. 